www. revis tad y o. com

\title{
Gamificación en Formación en Empresas
}

\author{
Gamification in Business Training
}

\author{
Estellés Miguel, $\mathrm{S}^{1}$, Rius Sorolla, $\mathrm{G}^{1}$, Palmer Gato, M.E ${ }^{1}$ and Albarracin Guillem, J.M ${ }^{1}$ \\ ${ }^{1}$ Dpto. de Organización de Empresas. Universitat Politècnica de Valencia. Edificio 7D. Camino de Vera s/n, 46022 Valencia. \\ soesmi@omp.upv.es,griuso@upv.es, marpalga@doe.upv.es, jmalbarr@omp.upv.es
}

Fecha de recepción: 6 marzo 2017

Fecha de aceptación: 5 julio 2017

Resumen: Se presenta la experiencia del uso de herramientas de gamificación, concretamente LEGO® Serious Play®, para la impartición de cursos, ventajas e inconvenientes. Esta herramienta ayuda en el desarrollo de la creatividad. En el caso de formación para empresas donde ellos ya lo han visto casi todo, es una manera de transformar el aprendizaje y servir como nexo de unión en el desarrollo de diferentes actividades. A continuación se expone la experiencia de los autores en el desarrollo de un análisis de debilidades y fortalezas del departamento comercial de una empresa de moda española con 104 tiendas en la península Ibérica.

Palabras clave: Gamificación, LEGO® Serious Play®, Innovación Educativa, formación en empresas.

Summary: This paper presents the introduction experience of gamification tools (in this case LEGO® Serious Play® -LSP-) in companies training and which are the advantages and disadvantages encountered with the use of this methodology. This tool helps in the development of the participants' creativity. In the case the participants have already seen almost everything, it is a way of transforming the class and serves as a union nexus in the development of different types of activities. Gamification is attracting interest in different areas. There are several studies that suggest that gamification will become a significant trend in the coming years. All games have four common traits: rules, a goal, a feedback and voluntary participation. With them is possible to create an attractive accession to the player. The game's dynamics are the motivation, effect and desire to pursue users and are based in turn on fundamental human needs. During the game, people are allowed to flow. The game brings together some of the most important features to learn: appeals to people motivation; you learn by participating (nobody can play for you) and error is an inherent part of the game. For all these reason, we decided to use the gamification in this experience. In this case, we selected LSP for the following advantages: 1) Promotes discovery itself generating commitment and trust with the problem, team, problem or company, 2) Involved participants as a part of a real team, 3) Facilitates the treatment of real situations in specific scenarios, learning to priorize and making decisions and 4) Allows the fast and simple way of diverse integration and complex factor. Below is exposed the experience of the authors in the analysis development of weaknesses and strenghs of the commercial department of a Spanish company selling fashion with more than 100 stores in Spain and Portugal. This experience has been conducted with the following objectives: 1) Proposing a different experience for teamwork, 2) Identify strengths and weaknesses of the commercial department of the company, 3) Encourage creativity when developing a SWOT, 4) Increasing teamwork participation. Perform: 1) Participants were divided into two groups around large work tables and were given in the middle lots of LEGO® bricks, 2) Each participant had to make a model with bricks to represent the idea they want to convey. Everyone could use sizes, shapes and colours as he deems necessary, 3) Then each participant explained it to their group (no one could criticize the others ideas), 4) At the end, the group must decide how to integrate the different models into one, putting the most important ideas in the centre and adding the rest in concentric circles, depending on its importance. Once the model is completed, each group explains their model. After making this experience, the conclusions are that all new activities are interesting, and arouse participation, provided they are well planned and conducted. We have met all the objectives in designing this activity. All participants have valued highly this activity, which they had never done before and they liked a lot. We have verified that it is really more participatory and the work grows better.

Keywords: Gamification, LEGO® Serious Play®, Educational Innovation, training in companies. 


\section{Introducción}

La gamificación es una práctica relativamente reciente que se puede situar entre los juegos, el marketing y la psicología, con ella se trata de crear experiencias atractivas y emocionantes para el usuario, empleando mecánicas del juego (Gallego y De Pablos, 2013).

Como Departamento de Organización de Empresas, tenemos bastante contacto con las empresas, en las que se nos suelen solicitar o bien formación en empresas o bien la colaboración en algún tipo de proyecto en las mismas. En este entorno, y aplicando la gamificación, el presente artículo presenta la utilización de LEGO $®$ Serious Play $®$ una herramienta de las denominadas de gamificación, en la impartición de cursos en Empresas y desarrollo de Planes Estratégicos.

En el presente caso nos vamos a centrar en la formación sobre DAFO (Debilidades, Amenazas, Fortalezas y Oportunidades), como paso previo al desarrollo de un Plan Estratégico de Empresa, y en la elaboración de uno mediante la utilización de esta herramienta en una empresa de moda con más de 100 tiendas a nivel nacional y con expansión en Portugal. Aunque en la presente experiencia sólo se realizo la parte de debilidades y fortalezas.

La experiencia se realizó con las 10 gerentes de zona de las distintas tiendas. En este artículo se describe la experiencia, para lo que se tiene en cuenta: que se busca con la misma, problemas que han aparecido en la realización y como se han solucionado. Por lo que se debe ver el diseño de la experiencia, los objetivos, la herramienta, problemas, etc. También se recogen los resultados de dicha experiencia y se relatan las conclusiones.

La estructura del presente artículo es la siguiente una introducción (punto 1), un apartado que presenta la herramienta a utilizar (LEGO $®$ Serious Play $®)$, en dicho apartado se presentan también los objetivos y la metodología (punto 2), un apartado en el que se recogen los resultados (punto 3 ) y finalmente uno que recoge las conclusiones (punto 4).

\section{Gamificación y LEGO $®$ Seri- ous Play®}

El término gamificación aparece en 2008 y a partir de este momento ha ido evolucionando su definición y es un concepto emergente en las disciplinas académicas (Estelles et al, 2014). La definición más utilizada es la de Deterding et al (2011) donde se define como "El uso de elementos de juego diseñados para contextos que no reúnen la naturaleza de juegos" (Ibíd, 2426), también en ese mismo año Cortizo et al (2011) lo define como "el proceso de diseñar experiencias divertidas para los usuarios dentro de contextos que no reúnen la naturaleza de juegos a partir de la aplicación de mecánicas de juegos y diseño de experiencias". Otra definición es "la utilización de mecánicas, estética y pensamiento de juegos para involucrar y comprometer personas, motivarlos hacia la acción, fomentando el aprendizaje y la resolución de problemas" (Kapp, 2012) y una de las que más se ajusta al presente caso sería "el uso de elementos estratégicos del diseño, pensamiento y mecánica de los juegos dentro de entornos que no reúnen en si mismos la naturaleza de juegos" (Raftopoulos, 2014). Según Mc. Gonigal (2011) todos los juegos tienen cuatro rasgos comunes: un objetivo o meta, reglas, un sistema de retroalimentación y la participación es voluntaria. Con estos rasgos se consigue crear una experiencia fácil y atractiva para el jugador.

También hay que tener en cuenta a la hora de hablar del juego las motivaciones intrínsecas y extrínsecas del jugador. La primera es la que lleva al jugador a realizar una actividad por propio placer, ya que es interesante y satisfactoria, la segunda es determinada por el entorno y su objetivo es obtener una meta externa (Amabile, 1996). No podemos olvidar que muchas cosas que consideramos "naturales" lo son ya que provienen de nuestra propia cultura (Oltra et al, 2017).

En esta línea aparece el LEGO $®$ Serious Play $\AA$ (LSP), que es una metodología de trabajo en equipo, comunicación, colaboración y resolución de problemas. El presidente de la empresa LEGO, no estaba satisfecho de los resultados de sus sesiones de estrategia y contacto con una universidad la IMD Business School para buscar una solución a dicho problema, con esto comenzó el desarrollo de lo que posteriormente se conocería como LSP, donde se vió la eficiencia con la que el conocimiento inconsciente pasa a ser consciente. 
Desde 2001, más de 200 empresas han utilizado LSP (Considiom, 2012) y la encuentran una metodología muy versátil. Es una herramienta de comunicación y un método de resolución de problemas basado en el uso del mayor éxito de ventas de los juegos de construcción. A través de las preguntas del facilitador cada participante construye un modelo propio, los modelos sirven de base para la toma de decisiones consensuadas.

Se marca un objetivo y los participantes se lanzan a la acción para resolver el desafio planteado. Todos los participantes de forma individual o colectiva, una vez escenifican su modelo pueden simular cambios que permiten visionar futuros cambios y sus posibles consecuencias dentro de la organización (Estellés et al, 2014).

\section{Cuando aplicar LSP:}

- $\quad$ En retos complejos y sin respuestas claras.

- $\quad$ Si existe necesidad de entender el conjunto, ver las conexiones.

- Si se pueden explorar potenciales soluciones.

- $\quad$ Diversidad de perfiles dentro de los participantes (en formación, edad, profesión, etc....).

- Para tomar decisiones que generen compromiso.

\subsection{Objetivos}

La experiencia se realizo con los siguientes objetivos:

1. Realizar una experiencia en grupo diferente.

2. Identificar las debilidades y fortalezas de la empresa.

3. Fomentar la creatividad de loas gerentes de área.

4. Preparar a loas gerentes de área para un trabajo colaborativo, creativo y participativo.

5. Qué loas gerentes de área vean que se tienen en cuenta sus aportaciones.

6. Que vean e interioricen las sinergias, es decir, como el trabajo en equipo puede superar los resultados del trabajo individual.

\subsection{Metodología}

Según la metodología LSP al construir con las manos algunos conceptos abstractos es posible tener acceso directo a conocimientos y experiencias almacenadas previamente en nuestra mente, esto facilita el razonamiento y la representación de situaciones, ideas, planes o proyectos. Las dinámicas del juego son la motivación, el deseo y el efecto que persiguen los que los utilizan y que al mismo tiempo se basan en las necesidades fundamentales del ser humano (Maslow, 1943, Reiss, 2004, Estelles et al, 2014).

Mediante LSP los participantes construyen modelos que representan de manera metafórica la percepción, motivación y sentimientos que tienen sobre un concepto determinado. Las piezas sirven como un lenguaje común que todos pueden usar, de forma que las ideas y opiniones de todos sean tomadas en cuenta. Por otro lado, se aconseja utilizar un conjunto de métodos que compartan la involucración del alumno en tareas del tipo: trabajo en equipo, análisis y síntesis. Desarrollando estrategias en las que además de actuar reflexionen sobre la acción que desarrollan (Hargreaves y Fink, 2003 y Estelles et al, 2013).

Los pasos serían los siguientes:

- $\quad$ Primero se explica el problema a resolver. En este caso hay que definir las debilidades de la empresa en la parte comercial. Posteriormente se verán las fortalezas, desde el punto de vista de los autores es interesante siempre finalizar la experiencia con la parte más positiva posible.

- $\quad$ Cada persona individualmente crea su propio modelo, así todos los participantes tienen tiempo de construir sus respuestas de forma individual. En esta parte cada participante deberá identificar las debilidades y fortalezas de la empresa en el aspecto comercial.

- $\quad$ Posteriormente, y con el modelo realizado cada persona explica al resto del grupo su visualización, historia y la metáfora que representa su montaje.

- $\quad$ No se pueden criticar las ideas del resto, todo aporta.

- A continuación entre todos los participantes deben crear un modelo unificado, en el que se represente en el centro lo más importante del modelo y hacia fuera lo menos importante, las decisiones aquí tomadas deben ser por consenso y se deben unificar todos los modelos individuales en un único modelo colectivo. En esta parte se fomenta la reflexión conjunta y el aprendizaje colaborativo. 
- $\quad$ Al terminar se lo explicaran al facilitador que es el que observa la experiencia y va anotando cambios significativos y la forma en la que se toman las decisiones de incorporar una parte u otra y los motivos. El facilitador, puede realizar preguntas durante la explicación del grupo si no entiende algo o para intentar extraer más información o incluso para comprobar si ha entendido perfectamente la misma.

\section{Resultados}

La actividad LSP ofrece una gran oportunidad de desarrollar la creatividad y la imaginación, de hecho se obtuvieron muchas ideas por parte de loas gerentes de área. Esta herramienta:

- $\quad$ Hace que los participantes se involucren, construyan de forma positiva, evitando problemas, crisis e interferencias de unos con otros, no permite la crítica por lo que se realiza un trabajo en equipo.

- Genera compromiso y confianza del equipo, promoviendo el descubrimiento del problema planteado y las posibles soluciones.

- $\quad$ Ayuda en la resolución de problemas reales en escenarios concretos.

- Integra factores muy diversos de resolución de problemas en un tiempo relativamente breve.

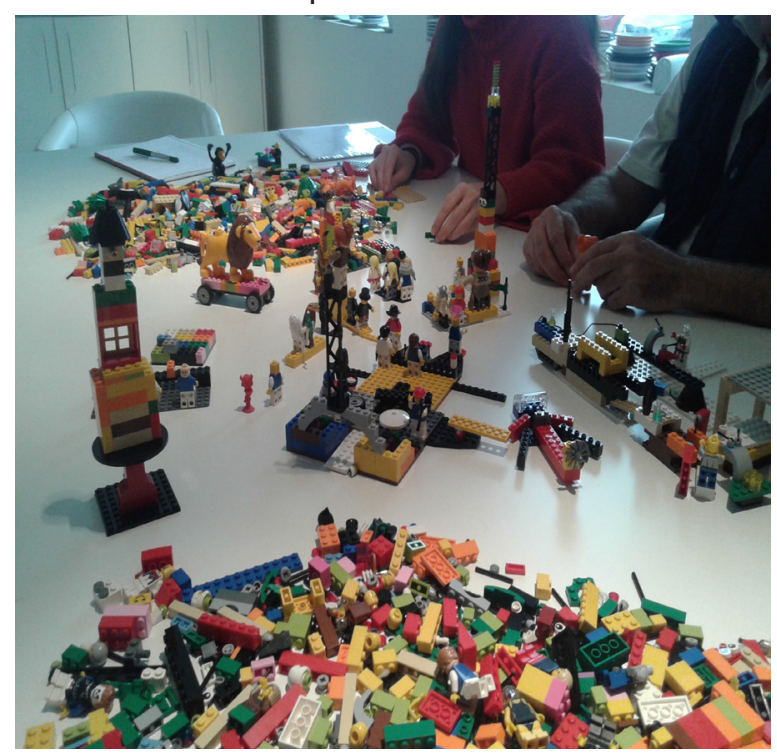

Fig. 1 Foto 1 de la sesión de trabajo. Fuente: Elaboración Propia.

Como el trabajo se realizó en una empresa privada no se puede incluir el resultado de la información extraída, pero se incluyen fotos de las sesiones.

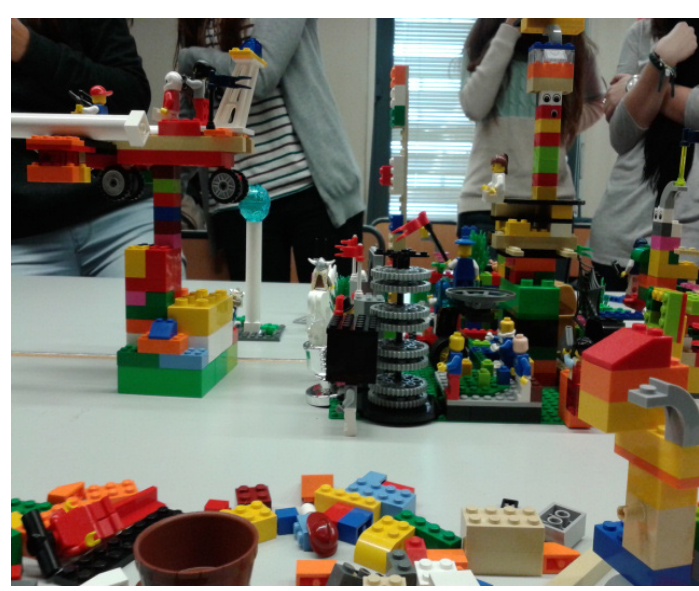

Fig. 2 Foto 2 de la sesión de trabajo. Fuente: Elaboración Propia.

Indicar que muchas de las ideas que se aportaron no habían aparecido hasta ese momento en otras sesiones de estrategia de la empresa, es decir, se consideraron totalmente innovadoras. Según la empresa, no solo eso, sino que fue la sesión estratégica realizada hasta el momento en la que más ideas se habían obtenido.

Indicar que en este caso en concreto y posiblemente por el sector empresarial en el que se desarrolla la actividad, los participantes fueron todo mujeres, excepto un hombre.

Las ventajas encontradas con el uso de LSP son: beneficio emocional (ayuda a liberar emociones), ayuda a desarrollar la creatividad, da seguridad ante los retos, mejora la concentración, desarrolla el razonamiento, da conciencia espacial y matemática.

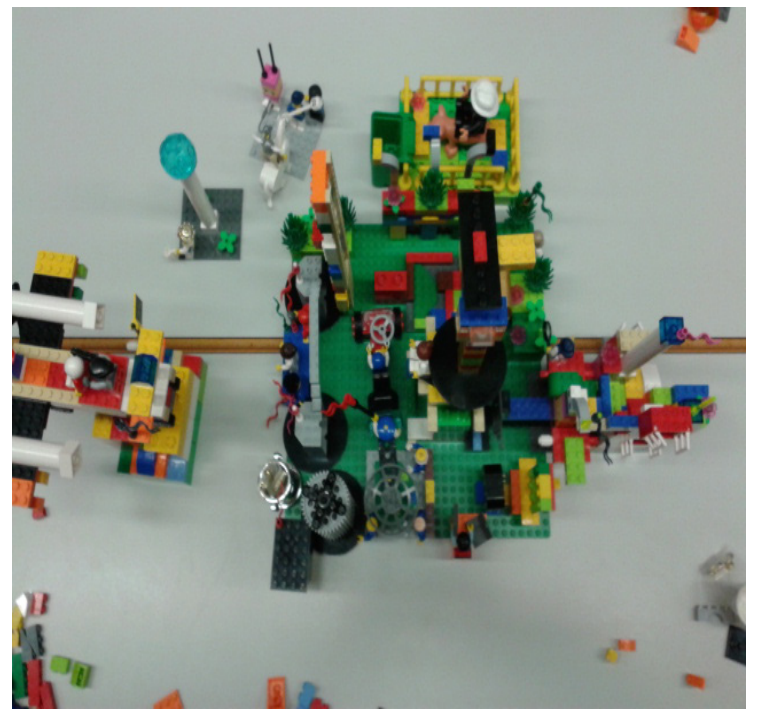

Fig. 3 Foto 3 de la sesión de trabajo. Fuente: Elaboración Propia. 
Como desventajas de momento sólo le hemos encontrado el precio, y la necesidad de un gran espacio y una gran mesa para poder realizar la actividad y finalmente el tener que trasladar cajones y cajones de piezas al lugar donde se vaya a desarrollar la actividad (para lo cuál nos hemos hecho con un carro).

\section{Conclusiones}

Cualquier experiencia innovadora es interesante, siempre que esté bien realizada, es decir que haya sido correctamente planificada y llevada a cabo.

Por otra parte se han conseguido todos los objetivos planteados en el apartado correspondiente, es decir, se ha realizado una experiencia de trabajo en grupo diferente, se han conseguido identificar las debilidades y fortalezas de la parte comercial de la empresa (aunque aquí no se exponen por temas de confidencialidad), se ha fomentado la creatividad de los participantes, que a su vez han realizado un trabajo colaborativo y participativo, se han tenido en cuenta las aportaciones de todos los participantes y ellos mismos han decido la importancia de las aportaciones y han comprendido la importancia de las sinergias en este tipo de actuaciones.

Todos los participantes en la experiencia la han valorado muy positivamente, ninguno de todos los participantes la había realizado con anterioridad y les ha parecido interesante y enriquecedora.

Dentro de una empresa, además de para lo que se ha utilizado en esta ocasión, se puede utilizar para: el diseño de nuevos productos y/o servicios, el desarrollo de la innovación dentro de la empresa, para el desarrollo de competencias, para promover la toma de decisiones colaborativa, para mejorar el trabajo en equipo cooperativo y colaborativo, mejorar la comunicación dentro de la organización, etc...

Lo que también hay que dejar claro es que no es solo un juego, no se comunica solo en una dirección, y no sirve solo para romper el hielo es algo más.

La gestión del tiempo es una parte importante de la experiencia. Por otro lado es un proceso que requiere de esfuerzo, tiempo, cooperación, colaboración y compromiso por parte de todos los miembros del equipo.

\section{References}

AMABILE, T. (1996). Creativity in Context: Update to the Social Psychology of Creativity. Boulder, CO, US. Westview Press.

CONSIDIOM (2012). La Historia de Lego Serious Play. http://considiom.com/blog/2012/01/la-historia-de-lego-serious-play/ Publicado (29/01/2012). Recuperado $(25 / 10 / 2017)$.

CORTIZO PÉREZ JC, CARRERO GARCÍA FM, MONSALVE PIQUERAS B, VELASCO COLLADO A, DEL DEDO LI, PÉREZ MARTíN J et al (2011). Gamificación y Docencia: Lo que la Universidad tiene que aprender de los videojuegos. VIII Jornadas Internacionales de Innovación Universitaria. Universidad Europea de Madrid.

DETERDING S, SICART M, NACKE L, O'HARA K \& DIXON D (2011). Gamification. Using game-design elements in non-gaming contexts. Proceedings of the 2011 annual conference extended abstracts on Human factors in computing system (pp 2425): New York. USA. ACM Press.

ESTELLES-MIGUEL S, BARBERA RIBERA MT ALBARRACÍN GUILLEM JM, PALMER GATO, ME (2014) Utilización del LEGO® Serious Play ${ }^{\circledR}$ en Clases de Marketing en Empresas de Servicios. INRED- 2014. Jornadas de Innovación Educativa y de Docencia en la Universitat Politècnica de València, pp 630-639.

ESTELLES-MIGUEL S, BARBERA RIBERA MT Y DEMA PÉREZ, CM. (2013) Experiencia de desarrollo de la competencia de trabajo en equipo. INNODOCT 2013. International Conference on Innovation, Documentation and Teaching Technologies, pp 528-532.

GALLEGO GOMEZ C \& DE PABLOS HEREDERO C (2013). The gamification ande the enrichment of innovatin practices in the firm: an analysis of experiences. Intangible Capital, vol 9, n.3, pp 800-822. http://doi.org/10.3926/ic.377

HARGREAVES, A. Y FINK, D. (2003). Estrategias de cambio y mejora en educación caracterizadas por su relevancia, difusión y continuidad en el tiempo. http://www.revistaeducacion.mec.es/re339/ re339_04.pdf 
KAPP KM (2012) The gamification of learning and instruction: game-based methods and strategies for training and educaction. John Wiley \& Sons.

MC.GONIGAL, J. (2011). Reality is Broken. Why Games Make us Better and How the Can Change the World. London. Penguin Press, H.C.

MASLOW AH (1943) A theory of human motivation en Psychological Review, vol. 50, n4, pp. 370-396.

OLTRA GUTIERREZ, J.V; GARRIGOS-SIMON, F.J. Y ESTELLES-MIGUEL, S. (2017) La ética como puerta a la informática en la educación primaria. Education in the Knowledge Society (EKS), Vol. 18, $n^{\circ} 2$, pp. 71-84. https://doi.org/10.14201/eks20171827183
RAFTOPOULOS M. (2014). Towards gamification transparency: A conceptual framework for the development of responsible gamified enterprise systems. Journal of Gaming \& Virtual Worlds, 6(2), 159-178. http://doi.org/10.1386/jgvw.6.2.159_1

REISS S (2004) Multifaceted nature of intrinsic motivation: The theory of 16 basic desires en Review of General Psychology, vol. 8, n.3, pp. 179-193. 